58) Culex univittatus Theobald, 1901.

59) Culex theileri Theobald, 1903.

60) Culex laticinctus Edwards, 1913.

61) Culex mimeticus Noé, 1899.

62) Culex brumpti Galliard, 1931.

J. Callot et J.-A. Rioux.

Institut de Parasitologie de la Faculté de Médecine de Strasbourg [Bas-Rhin, France] et laboratoire d'Ecologie médicale et de Pathologie parasitaire, Faculté de Médecine de Montpellier (Hérault)

\title{
Association Internationale pour l'Etude des Filarioses (International Filariosis Association)
}

L'Association Internationale pour l'étude des Filarioses est issue des groupes de travail réunis en 1955 et en 1961 sous les auspices de l'O.M.S. pour étudier l'onchocercose. Elle a été créée pour encourager l'étude des filarioses et l'organisation de campagnes de lutte contre les maladies.

La première Réunion Générale de l'Association a eu lieu à Rio de Janeiro le 8 septembre 1963, durant le Congrès International de Parasitologie. Les réunions ultérieures auront lieu en fonction des occasions offertes par les Congrès Internationaux.

Des mesures seront prises, chaque fois que ce sera possible, pour encourager l'étude de tous les types de filarioses en organisant des réunions régionales, et pour attirer officiellement l'attention des gouvernements et des organisations internationales sur les problèmes posés par les filarioses. L'Association facilitera l'échange d'informations techniques entre les spécialistes des filarioses et espère bénéficier de leur coopération.

L'Association possède un Conseil exécutif de 15 membres représentant les différentes régions du monde. Le Président est le Professeur J. F. Kessel (Etats-Unis). Les Vice-Présidents sont le Professeur P. C. C. Garnham (Royaume-Uni) et le Médecin Général Inspecteur P. Richet (France). MM. les Secrétaires sont le Docteur M. Giaquinto (Italie) et le Docteur F. Hawking * (Royaume-Uni) qui fourniront sur demande toute information complementaire concernant l'Association.

Cette Association Internationale serait heureuse de recevoir l'adhésion de tous ceux s'intéressant à l'étude et au contrôle des filarioses.

* National Institute for Medical Research, Mill Hill, London N.W.7, Angleterre. 